\title{
Acute Febrile Illness and Complications Due to Murine Typhus, Texas, USA ${ }^{1,2}$
}

\author{
Zeeshan Afzal, Sunand Kallumadanda, Feng Wang, Vagish Hemmige, Daniel Musher
}

Murine typhus occurs relatively commonly in southern Texas, as well as in California. We reviewed records of 90 adults and children in whom murine typhus was diagnosed during a 3-year period in 2 hospitals in southern Texas, USA. Most patients lacked notable comorbidities; all were immunocompetent. Initial signs and symptoms included fever (99\%), malaise $(82 \%)$, headache $(77 \%)$, fatigue $(70 \%)$, myalgias $(68 \%)$, and rash $(39 \%)$. Complications, often severe, in $28 \%$ of patients included bronchiolitis, pneumonia, meningitis, septic shock, cholecystitis, pancreatitis, myositis, and rhabdomyolysis; the last 3 are previously unreported in murine typhus. Low serum albumin and elevated procalcitonin, consistent with bacterial sepsis, were observed in $>70 \%$ of cases. Rash was more common in children; thrombocytopenia, hyponatremia, elevated hepatic transaminases, and complications were more frequent in adults. Murine typhus should be considered as a diagnostic possibility in cases of acute febrile illness in southern and even in more northern US states.

$\mathrm{M}$ urine (endemic) typhus is most frequently recognized as an acute febrile illness, although the clinical manifestations of this infection cover the full spectrum of disease, from asymptomatic infection to fulminant disease and death (1-5). The causative organism, Rickettsia typhi, is a small, gram-negative, obligate intracellular bacterium. Worldwide, especially in tropical and subtropical areas, the roof rat (Rattus rattus) and Norway rat (Rattus norvegicus) have been the principal reservoirs, with the rat flea, Xenopsylla cheopis, as the principal vector $(2,6)$.

In the United States, individual murine typhus cases and outbreaks have been reported from suburban areas; in these instances, opossums (Didelphis virginiana) and Rattus spp. have been implicated as the reservoirs, with the cat flea, Ctenocephalides felis, as the principal vector (2). The incidence of murine typhus declined sharply in the United States after the institution of DDT for control

Author affiliations: University of Texas Rio Grande Valley, McAllen, Texas, USA (Z. Afzal, S. Kallumadanda, F. Wang); Baylor College of Medicine, Houston, Texas, USA (V. Hemmige, D. Musher); M.E. DeBakey VA Medical Center, Houston (D. Musher)

DOI: https://doi.org/10.3201/eid2308.161861 of rat fleas in 1945 (5), but it now appears to be on the rise, especially in southern Texas and California $(2,7,8)$. We describe 90 murine typhus infections that were diagnosed in 2 hospitals in Hidalgo County, Texas, USA, during a 3-year period, with a comparison of clinical manifestations in adults and children.

\section{Methods}

\section{Patient Identification}

We searched microbiology laboratory records at McAllen Medical Center (McAllen, TX, USA) and Edinburg Regional Medical Center (Edinburg, TX, USA) for July 1, 2013-June 30, 2016, to identify patients who had typhus group rickettsiae $\mathrm{IgM}$ or $\mathrm{IgG}$ at a titer $\geq 1: 128$ (9) (Focus Diagnostics Rickettsia, Indirect Immunofluorescence Antibody for IgM and IgG; Labcorp, Houston, TX, USA). A total of 101 patients were identified; all had been hospitalized. We carefully reviewed their medical records to be certain that their clinical syndrome was suggestive of murine typhus.

Eleven of the 101 patients were excluded: 5 had incomplete medical records sufficient to make a clinical diagnosis and 6 had another condition that might have caused a fever (otitis media, active hepatitis C, Epstein-Barr virus infection, untreated HIV infection, lymphoma, or tuberculosis). IgG titers had been measured in every case. Of the remaining 90 patients, $41(45.6 \%)$ had been evaluated by an infectious disease consultant, who either concurred with or suggested the diagnosis of murine typhus in every case.

\section{Case Definitions}

For case identification, we used the Flea-borne Typhus Epi Case Criteria 2016 Case Definition/Case Classification of the Texas State Department of Health (9). We also used the case classification scheme described by the

\footnotetext{
${ }^{1} \mathrm{~A}$ poster form of this study was presented at the Annual Session and Primary Care Summit of Texas Academy of Family Physicians, November 4-6, 2016, Dallas, TX, USA.
}

${ }^{2} \mathrm{An}$ abstract has been accepted for ID Week 2017, October 4-8, 2017, San Diego, CA, USA, for an oral presentation. 
Centers for Disease Control and Prevention for spotted fever rickettsiosis 2010 to categorize our patients with suspected murine typhus, probable murine typhus, or confirmed murine typhus (10). Patients with suspected murine typhus were those with laboratory evidence of past or present infection but without a clinically compatible illness. Patients with probable murine typhus were those in whom the clinical disease was compatible with murine typhus (meets clinical evidence criteria) and with supportive laboratory results. Patients with confirmed murine typhus had a clinically compatible illness and laboratory confirmation. Illnesses that are clinically compatible with murine typhus are characterized by acute onset of fever and $\geq 1$ : headache, myalgia, anorexia, nausea/vomiting, thrombocytopenia, or elevated hepatic aminotransferases. Supportive laboratory evidence is defined by indirect immunofluorescence assay (IgM or $\operatorname{IgG}$ ) serologic titer $\geq 1: 128$. Laboratory-confirmed cases are required to have serologic evidence of a 4-fold increase in IgG-specific antibody titer reactive with typhus group rickettsiae antigen by indirect immunofluorescence assay in paired serum specimens ( 1 taken in the first week of illness and a second 2-4 weeks later).

\section{Data Gathering}

We reviewed electronic medical records to obtain data on demography and epidemiology; comorbid conditions; symptoms and their duration before hospitalization; physical findings; laboratory results (hematology, chemistry, and microbiology); imaging; requirements for intensive care; hospital course; antimicrobial drug treatment; response to treatment; and duration of hospital stay. This study was approved by the institutional review board of the University of Texas Rio Grande Valley Medical School.

\section{Data Analysis}

Basic descriptive statistics were calculated, and comparisons between the proportion of adult and pediatric patients with different signs and symptoms of murine typhus were calculated with a $\chi^{2}$ or Fisher exact test, as appropriate. All analyses were performed in Stata 12 (Statacorp LLC, College Station, TX, USA).

\section{Results}

On the basis of the definitions we described, 90 patients were given a diagnosis of murine typhus. One patient had a clinical syndrome consistent with murine typhus but the presence of fever was not documented; this illness was characterized as suspected murine typhus. Eighty-six patients met criteria for probable murine typhus, and 3 had confirmed murine typhus. Overall, the initial IgM titer was $\geq 1: 128$ in $86(96 \%)$ cases, and the IgG titer was $\geq 1: 128$ in $63(70 \%)$ cases. Five patients had a second blood sample submitted for typhus group rickettsiae IgG; 3 of these exhibited a 4-fold increase in IgG titer (rising from $<1: 64$ to $1: 512$ ) and were determined to be confirmed murine typhus. One patient had a 2-fold increase in IgG titer; in the other patient, IgG titer remained unchanged but IgM titer doubled from 1:128 to $1: 256$, thus meeting the criteria for probable murine typhus.

Of the 90 patients, $36(40 \%)$ were $<18$ years of age, and $45(50 \%)$ were female. All patients included in the final analysis had a clinical syndrome suggestive of murine typhus (Table 1), including fever in $99 \%$, malaise in $82 \%$, headache in $77 \%$, fatigue in $70 \%$, myalgias in $68 \%$, and rash in $39 \%$. Other causes of the syndrome were sought by bacteriologic and serologic studies in a varying proportion of these cases; no other cause was found.

Complications of murine typhus occurred in 25 (28\%) cases. Among documented complications were bronchiolitis in 2 cases, pneumonia in 8 , pancreatitis in 3 , cholecystitis in 1 , myositis (creatine phosphokinase $>1,500 \mathrm{U} / \mathrm{L}$ ) in 1, rhabdomyolysis (creatine phosphokinase $>4,000 \mathrm{U} / \mathrm{L}$ ) in 2, meningitis in 2 , sepsis with acute kidney injury in 1, and septic shock in 4 . In 1 patient, pneumonia and septic shock occurred together. There were no deaths.

Laboratory findings consistent with murine typhus included leukocytes $<6,000$ cells $/ \mathrm{mm}^{3}$ in $21(23 \%)$ patients, platelets $<120,000 / \mathrm{mm}^{3}$ in $49(54 \%)$, aspartate aminotransferase $>50 \mathrm{IU} / \mathrm{L}$ in $76(84 \%)$, alanine aminotransferase $>50$ IU/L in $67(74 \%)$, and serum sodium $\leq 133 \mathrm{mmol} / \mathrm{L}$ in 40 (44\%). In no case was the leukocyte count as low as 3,000 cells $/ \mathrm{mm}^{3}$. Serum albumin was $<3.5 \mathrm{~g} / \mathrm{dL}$ in $73(81 \%)$ of 90 cases, and serum procalcitonin was $\geq 0.5 \mathrm{ng} / \mathrm{mL}$ in 10 (71\%) of 14 cases, both findings consistent with bacterial sepsis. Newly recognized complications of murine typhus in this case series included pancreatitis, myositis, and rhabdomyolysis. Thirteen patients $(14 \%)$ required care in the intensive care unit.

We found notable differences between adult and pediatric patients (Table 1) in the frequency of diarrhea, platelet count $<120,000 / \mathrm{mm}^{3}$, serum sodium $\leq 133 \mathrm{mmol} / \mathrm{L}$, and aspartate aminotransferase and alanine aminotransferase $>50$ $\mathrm{IU} / \mathrm{L}$, all of which were more common in adults. Complications occurred in $18(33 \%)$ of 54 adults and $7(19 \%)$ of 36 children $(\mathrm{p}=0.15)$.

The median duration from onset of illness to hospitalization was 7 days (range 1-21 days). Many patients saw a physician before coming to the hospital. We determined that the median time between the initial clinical encounter and institution of effective antimicrobial drug therapy was 5 days (range $0-15$ days). A wide array of antimicrobial drugs was prescribed. Eight patients never received an antimicrobial drug appropriate to treat murine typhus, but the disease resolved spontaneously. 
Table 1. Characteristics of 90 patients with murine typhus, Hidalgo County, Texas, USA, July 1, 2013-June 30, 2016*

\begin{tabular}{|c|c|c|c|c|}
\hline \multirow[b]{2}{*}{ Characteristic } & \multicolumn{4}{|c|}{ No. (\%) patients } \\
\hline & Age $\geq 18 y$ & Age $<18$ y & Total & p value \\
\hline \multicolumn{5}{|l|}{ Symptoms } \\
\hline Subjective fever/chills & $53 / 54(98)$ & $36 / 36(100)$ & $89 / 90(99)$ & 1 \\
\hline Malaise & $21 / 24(88)$ & $15 / 20(75)$ & $36 / 44(82)$ & 0.44 \\
\hline Headache & $37 / 46(80)$ & $23 / 32(72)$ & $60 / 78(77)$ & 0.38 \\
\hline Fatigue & $22 / 29(76)$ & $10 / 17(59)$ & $32 / 46(70)$ & 0.22 \\
\hline Myalgias & $29 / 39(74)$ & $13 / 23(57)$ & $42 / 62(68)$ & 0.15 \\
\hline Nausea and/or vomiting & $31 / 52(60)$ & $21 / 35(60)$ & $52 / 87(60)$ & 0.97 \\
\hline Cough & $21 / 52(40)$ & $18 / 36(50)$ & $39 / 88(44)$ & 0.37 \\
\hline Abdominal pain & $17 / 47(36)$ & 10/33 (30) & $27 / 80(34)$ & 0.58 \\
\hline Arthralgia & $9 / 34(26)$ & $4 / 24(17)$ & $13 / 58(22)$ & 0.53 \\
\hline Diarrhea & $9 / 50$ (18) & $1 / 35(3)$ & $10 / 85(12)$ & 0.04 \\
\hline Confusion & $6 / 42(14)$ & $2 / 30(7)$ & $8 / 72(11)$ & 0.45 \\
\hline \multicolumn{5}{|l|}{ Signs } \\
\hline Temperature $\geq 100.4^{\circ} \mathrm{F}$ & $48 / 54(89)$ & 34/36 (94) & $82 / 90(91)$ & 0.47 \\
\hline Rash & $16 / 51(31)$ & $18 / 36(50)$ & $34 / 87(39)$ & 0.12 \\
\hline Enlarged liver & $1 / 44(2)$ & $1 / 32(3)$ & $2 / 76(3)$ & 1 \\
\hline Enlarged spleen & $0 / 44(0)$ & $1 / 32(3)$ & $1 / 76(1)$ & 0.42 \\
\hline \multicolumn{5}{|l|}{ Laboratory findings } \\
\hline Leukocytes $<6,000$ cells $/ \mathrm{mm}^{3}$ & $10 / 54(19)$ & $11 / 36(31)$ & $21 / 90(23)$ & 0.19 \\
\hline Leukocytes $<3,000$ cells $/ \mathrm{mm}^{3}$ & 0 & 0 & 0 & 1 \\
\hline Platelets $<120,000 / \mathrm{mm}^{3}$ & $37 / 54(69)$ & $12 / 36(33)$ & $49 / 90(54)$ & $<0.01$ \\
\hline Bilirubin $\geq 1.5 \mathrm{mg} / \mathrm{dL}$ & $14 / 54(26)$ & $3 / 36(8)$ & $17 / 90(19)$ & 0.05 \\
\hline AST $>50 \mathrm{IU} / \mathrm{L}$ & $51 / 54(94)$ & $25 / 36(69)$ & $76 / 90(84)$ & $<0.01$ \\
\hline $\mathrm{ALT}>50 \mathrm{IU} / \mathrm{L}$ & $45 / 54(83)$ & $22 / 36(61)$ & $67 / 90(74)$ & 0.02 \\
\hline Serum sodium $\leq 133 \mathrm{mmol} / \mathrm{L}$ & $34 / 54(63)$ & $6 / 36(17)$ & $40 / 90(44)$ & $<0.01$ \\
\hline Serum albumin $<3.5 \mathrm{~g} / \mathrm{dL}$ & $46 / 54(85)$ & $27 / 36(75)$ & $73 / 90(81)$ & 0.23 \\
\hline Serum procalcitonin $>0.5 \mathrm{ng} / \mathrm{mL}$ & $9 / 13(69)$ & $1 / 1(100)$ & $10 / 14(71)$ & 1 \\
\hline $\lg M \geq 1: 128$ & $50 / 54(93)$ & $36 / 36(100)$ & $86 / 90(96)$ & 0.15 \\
\hline $\lg G \geq 1: 128$ & $41 / 54(76)$ & $22 / 36(61)$ & $63 / 90(70)$ & 0.13 \\
\hline Complications & $18 / 54(33)$ & $7 / 36(19)$ & $25 / 90(28)$ & 0.15 \\
\hline
\end{tabular}

\section{Discussion}

Murine typhus causes a syndrome characterized by nonspecific manifestations, including fever, headache, myalgias, malaise, nausea, and vomiting in more than half of cases. This syndrome has previously been described throughout the world, including the southwestern United States $(1,4,5,11-13)$ and California $(2,14)$, as well as Europe $(3,15-17)$.

Twenty-three of the 90 patients in this study were first seen in a primary care physician's office. Diagnoses made at the time of that initial encounter, whether in children or adults, included viral syndrome, influenza, and streptococcal pharyngitis; murine typhus was not considered in any of them. Once patients were hospitalized, this nonspecific clinical syndrome, together with characteristic laboratory abnormalities (low leukocyte and platelet counts and elevated levels of liver enzymes) and knowledge of the occurrence of murine typhus in this area, enabled the correct diagnosis to be considered. Serum samples were submitted for supportive laboratory testing, and appropriate therapy was instituted promptly, in most cases before results of serologic tests were known. It is still worth noting that, for 8 patients, the diagnosis of murine typhus was never made during their illness, but only when serologic results were returned. Illness resolved spontaneously in these 8 patients.
Serum procalcitonin, not previously reported in murine typhus, was elevated in 10 (71.4\%) of the 14 cases in which it was studied; this test might help distinguish murine typhus from a viral syndrome in an endemic area. The low serum albumin is also consistent with bacterial sepsis (18).

Although the usual symptoms were those of a nonspecific illness suggestive of influenza, another viral syndrome, or streptococcal pharyngitis, many of our patients were, at some point, quite ill. Thirteen (14\%) of 90 were hospitalized in an intensive care unit. Six met criteria for sepsis; serum albumin fell to $<3.5 \mathrm{~g} / \mathrm{dL}$, consistent with serious bacterial infection, in $73(81 \%)$ of the 90 patients. Complications of murine typhus, defined as symptoms, signs, or both beyond nonspecific ones, were seen in 25 $(28 \%)$ patients and appeared more frequently in adults than in children (Table 2). The most common complications were pulmonary complications, such as bronchiolitis and pneumonia; aseptic meningitis; and sepsis with shock. These complications have been described previously, either in case reports or in case series $(1,2,12,16,17,19)$. Only 2 previous studies have specifically tabulated complications $(3,20)$, with the frequency of complications in 1 prospective series being similar to that in this study (20).

No previous investigation has reported on serum procalcitonin, which was elevated in $10(71.4 \%)$ of 14 patients in the study in which it was measured. Newly described 
Table 2. Complications in 90 patients with murine typhus, Hidalgo County, Texas, USA, July 1, 2013-June 30, 2016

\begin{tabular}{lccc}
\hline Complication & $\begin{array}{c}\text { Age } \geq 18 \mathrm{y}, \\
\mathrm{n}=54\end{array}$ & $\begin{array}{c}\text { Age }<18 \mathrm{y}, \\
\mathrm{n}=36\end{array}$ & $\begin{array}{c}\text { Total, } \\
\mathrm{n}=90\end{array}$ \\
\hline Bronchiolitis & 0 & 2 & 2 \\
Pneumonia & 7 & 1 & 8 \\
Pancreatitis & 3 & 0 & 3 \\
Cholecystitis & 0 & 1 & 1 \\
Myositis & 0 & 1 & 1 \\
Rhabdomyolysis & 0 & 2 & 2 \\
Meningitis & 2 & 0 & 2 \\
Septic shock & 4 & 0 & 4 \\
Sepsis with acute & 1 & 0 & 1 \\
$\quad$ kidney injury & & & \\
Pneumonia plus septic shock & 1 & 0 & 1 \\
\hline Total & 18 & 7 & 25 \\
\hline
\end{tabular}

complications in this series included pancreatitis, myositis, and rhabdomyolysis. Unusual cutaneous manifestations (21), iritis (20), lymphadenopathy (20), splenic rupture $(22,23)$, disseminated intravascular coagulation $(3,12)$, cholecystitis (24), and endocarditis $(25,26)$ have previously been reported; except for 1 patient with cholecystitis, we had no examples of these other unusual complications in our series.

Earlier series of cases have not compared findings in adults and children. Differences between children and adults in this study included greater frequency of rash in children, whereas statistically significant differences were greater incidence of diarrhea, thrombocytopenia, hyponatremia, and elevated hepatic aminotransferases in adults. Complicated illness was more common in older than in younger subjects, as has been suggested in separate reports from a group of investigators in Greece $(16,17)$.

Murine typhus has been increasingly recognized in Texas (8). Two counties in Texas have reported $>50$ cases of murine typhus per year $(1,11)$, and in 1 of these, a high prevalence of antibodies to $R$. typhi has been described in healthy children (13). To our knowledge, such a study has not been reported in adults in southern Texas, the area of greatest endemicity in the United States. Our finding of 90 recognized cases of murine typhus in 2 hospitals in a 3 -year period attests to the frequency of murine typhus in the southwestern United States.

Rats and opossums that coexist in urban and suburban settings appear to be reservoirs for the organism. The vector has traditionally been thought to be Xenopsylla cheopis, the rat flea (6). However, recent studies $(27,28)$ have shown that the cat flea, Ctenocephalides felis, which also infests opossums, plays a role in transmission. Blanton et al. (27) found rickettsial DNA in pools of fleas obtained from opossums in Galveston, Texas, and documented the emergence of antibodies to Rickettsia sp. in these animals. A major outbreak of murine typhus in Austin, Texas, was traced to contact with opossums (11). Because R. felis may also be present in fleas living on these same reservoirs $(29,30)$ and the clinical spectrum of infection due to this organism remains undefined, it is possible that some cases attributed to $R$. typhi and identified as murine typhus may actually be due to $R$. felis.

The natural habitat of the American opossum includes northeastern and northwestern states, suggesting the possibility that murine typhus may not be confined to areas that are currently recognized as endemic, such as southern Texas or California. In Virginia and the Carolinas, illnesses of patients with fever, rash, and nonspecific symptoms are regularly diagnosed as Rocky Mountain spotted fever and treated with doxycycline; however, it is possible that some of these infections may be due to R. typhi. PCR $(24,31,32)$ can distinguish among these organisms, but it is not readily available and has uncertain clinical value. $R$. felis may also cause human infection and requires PCR to distinguish it from $R$. typhi $(29,30,33)$. In fact, as suggested by Eremeeva et al. (29), murine typhus as we now know it may be a single clinical syndrome that can be caused by either $R$. typhi or R. felis.

The principal limitation of our study is that diagnoses were suspected clinically but were supported by detection of serum antibodies reactive with typhus group rickettsial antigen. Cross-reacting IgM may also appear after infection with other organisms in the typhus group ( $R$. prowazecki) and the spotted fever group ( $R$. rickettsii, the cause of Rocky Mountain spotted fever), as well as R. felis; we cannot exclude the possibility that some of our cases may have been caused by these organisms. Other cases of $R$. typhi infection may have been missed because physicians suspected the disease very early and instituted treatment with doxycycline. The principal strengths of this study are that the medical records were reviewed in every case by the principal investigator and that many of the patients had been seen by an infectious disease consultant who concurred with the final diagnosis, usually before serologic results were available.

In conclusion, murine typhus is a multifaceted disease that is common in southern Texas and California and that might be identified more frequently in other areas of the United States if the diagnosis were sought. A major description of this disease, especially with newly proposed case definitions, has not appeared in an American journal for 15 years; pediatric and adult cases have not been compared, and the range of complications in the United States has not been described recently. The population of the American opossum has increased in northern states. In Texas and California, their fleas are known to carry R. typhi $(27,34)$ but, to our knowledge, a search for Rickettsia spp. in opossums has not been conducted in other parts of the United States. We suggest that, in cases of acute febrile conditions, the diagnosis of murine typhus needs to be considered elsewhere in the southern United 
States and, perhaps, farther north as well. Because of the high prevalence of murine typhus in areas of recognized endemicity, medical centers might consider on-site testing for antibodies to typhus group rickettsiae to support a clinical decision and early treatment. A reactive serology might then trigger a reflex request to obtain convalescent titers to provide confirmatory data.

Dr. Afzal is a third-year family medicine resident and serves as assistant chief resident at the McAllen family medicine residency program, University of Texas Rio Grande Valley, McAllen, Texas. He is interested in clinical research and rural health. Dr. Musher is a distinguished service professor of medicine and professor of molecular virology and microbiology at Baylor College of Medicine in Houston, Texas.

\section{References}

1. Whiteford SF, Taylor JP, Dumler JS. Clinical, laboratory, and epidemiologic features of murine typhus in 97 Texas children. Arch Pediatr Adolesc Med. 2001;155:396-400. http://dx.doi.org/ 10.1001/archpedi.155.3.396

2. Civen R, Ngo V. Murine typhus: an unrecognized suburban vectorborne disease. Clin Infect Dis. 2008;46:913-8. http://dx.doi.org/10.1086/527443

3. Chaliotis G, Kritsotakis EI, Psaroulaki A, Tselentis Y, Gikas A. Murine typhus in central Greece: epidemiological, clinical, laboratory, and therapeutic-response features of 90 cases. Int J Infect Dis. 2012;16:e591-6. http://dx.doi.org/10.1016/ j.ijid.2012.03.010

4. Taylor JP, Betz TG, Rawlings JA. Epidemiology of murine typhus in Texas. 1980 through 1984. JAMA. 1986;255:2173-6. http://dx.doi.org/10.1001/jama.1986.03370160071029

5. Older JJ. The epidemiology of murine typhus in Texas, 1969. JAMA. 1970;214:2011-7. http://dx.doi.org/10.1001/ jama.1970.03180110021004

6. Azad AF. Epidemiology of murine typhus. Annu Rev Entomol. 1990;35:553-69. http://dx.doi.org/10.1146/annurev.en.35.010190. 003005

7. Texas Department of State Health Services. Murine typhus [cited 2016 Aug 15]. http://www.dshs.texas.gov/idcu/disease/ murine_typhus/

8. Blanton LS, Vohra RF, Bouyer DH, Walker DH. Reemergence of murine typhus in Galveston, Texas, USA, 2013. Emerg Infect Dis. 2015;21:484-6. http://dx.doi.org/10.3201/eid2103.140716

9. Mayes B. Rickettsial disease diagnostics and epidemiology [cited 2017 Mar 14]. https://dshs.texas.gov/WorkArea/linkit.aspx? LinkIdentifier $=$ id\&ItemID $=8590006123$

10. Centers for Disease Control and Prevention. Spotted fever rickettsiosis, case definition 2010 [cited 2017 Mar 14]. https://wwwn.cdc.gov/nndss/conditions/spotted-fever-rickettsiosis/ case-definition/2010/

11. Adjemian J, Parks S, McElroy K, Campbell J, Eremeeva ME, Nicholson WL, et al. Murine typhus in Austin, Texas, USA, 2008. Emerg Infect Dis. 2010;16:412-7. http://dx.doi.org/10.3201/ eid1603.091028

12. Dumler JS, Taylor JP, Walker DH. Clinical and laboratory features of murine typhus in south Texas, 1980 through 1987. JAMA. 1991;266:1365-70. http://dx.doi.org/10.1001/ jama.1991.03470100057033

13. Purcell K, Fergie J, Richman K, Rocha L. Murine typhus in children, south Texas. Emerg Infect Dis. 2007;13:926-7. http://dx.doi.org/10.3201/eid1306.061566
14. Green JS, Singh J, Cheung M, Adler-Shohet FC, Ashouri N. A cluster of pediatric endemic typhus cases in Orange County, California. Pediatr Infect Dis J. 2011;30:163-5. http://dx.doi.org/ 10.1097/INF.0b013e3181f4cc25

15. Bernabeu-Wittel M, Pachón J, Alarcón A, López-Cortés LF, Viciana P, Jiménez-Mejías ME, et al. Murine typhus as a common cause of fever of intermediate duration: a 17-year study in the south of Spain. Arch Intern Med. 1999;159:872-6. http://dx.doi.org/10.1001/archinte.159.8.872

16. Gikas A, Kokkini S, Tsioutis C, Athenessopoulos D, Balomenaki E, Blasak S, et al. Murine typhus in children: clinical and laboratory features from 41 cases in Crete, Greece. Clin Microbiol Infect. 2009;15(Suppl 2):211-2. http://dx.doi.org/10.1111/ j.1469-0691.2008.02133.x

17. Tsioutis C, Chaliotis G, Kokkini S, Doukakis S, Tselentis Y, Psaroulaki A, et al. Murine typhus in elderly patients: a prospective study of 49 patients. Scand J Infect Dis. 2014;46:779-82. http://dx.doi.org/10.3109/00365548.2014.943283

18. Fleck A, Hawker F, Wallace PI, Raines G, Trotter J, Ledingham IM, et al. Increased vascular permeability: a major cause of hypoalbuminaemia in disease and injury. Lancet. 1985;325:781-4. http://dx.doi.org/10.1016/S0140-6736(85)91447-3

19. Bernabeu-Wittel M, Villanueva-Marcos JL, Alarcón-González A, Pachón J. Septic shock and multiorganic failure in murine typhus. Eur J Clin Microbiol Infect Dis. 1998;17:131-2. http://dx.doi.org/10.1007/BF01682172

20. Gikas A, Doukakis S, Pediaditis J, Kastanakis S, Psaroulaki A, Tselentis Y. Murine typhus in Greece: epidemiological, clinical, and therapeutic data from 83 cases. Trans R Soc Trop Med Hyg. 2002;96:250-3. http://dx.doi.org/10.1016/S0035-9203(02)90090-8

21. Blanton LS, Lea AS, Kelly BC, Walker DH. An unusual cutaneous manifestation in a patient with murine typhus. Am J Trop Med Hyg. 2015;93:1164-7. http://dx.doi.org/10.4269/ajtmh.15-0380

22. Fergie J, Purcell K. Spontaneous splenic rupture in a child with murine typhus. Pediatr Infect Dis J. 2004;23:1171-2.

23. McKelvey SD, Braidey PC, Stansby GP, Weir WRC. Spontaneous splenic rupture associated with murine typhus. J Infect. 1991;22:296-7. http://dx.doi.org/10.1016/S0163-4453(05)80017-9

24. Schriefer ME, Sacci JB Jr, Dumler JS, Bullen MG, Azad AF. Identification of a novel rickettsial infection in a patient diagnosed with murine typhus. J Clin Microbiol. 1994;32:949-54.

25. Austin SM, Smith SM, Co B, Coppel IG, Johnson JE. Serologic evidence of acute murine typhus infection in a patient with culture-negative endocarditis. Am J Med Sci. 1987;293:320-3. http://dx.doi.org/10.1097/00000441-198705000-00007

26. Buchs AE, Zimlichman R, Sikuler E, Goldfarb B. Murine typhus endocarditis. South Med J. 1992;85:751-3. http://dx.doi.org/10.1097/00007611-199207000-00019

27. Blanton LS, Idowu BM, Tatsch TN, Henderson JM, Bouyer DH, Walker DH. Opossums and cat fleas: new insights in the ecology of murine typhus in Galveston, Texas. Am J Trop Med Hyg. 2016;95:457-61. http://dx.doi.org/10.4269/ajtmh.16-0197

28. Karpathy SE, Hayes EK, Williams AM, Hu R, Krueger L, Bennett S, et al. Detection of Rickettsia felis and Rickettsia typhi in an area of California endemic for murine typhus. Clin Microbiol Infect. 2009;15(Suppl 2):218-9. http://dx.doi.org/10.1111/ j.1469-0691.2008.02140.x

29. Eremeeva ME, Karpathy SE, Krueger L, Hayes EK, Williams AM, Zaldivar Y, et al. Two pathogens and one disease: detection and identification of flea-borne Rickettsiae in areas endemic for murine typhus in California. J Med Entomol. 2012;49:1485-94. http://dx.doi.org/10.1603/ME11291

30. Abramowicz KF, Rood MP, Krueger L, Eremeeva ME. Urban focus of Rickettsia typhi and Rickettsia felis in Los Angeles, California. Vector Borne Zoonotic Dis. 2011;11:979-84. http://dx.doi.org/10.1089/vbz.2010.0117 
31. Henry KM, Jiang J, Rozmajzl PJ, Azad AF, Macaluso KR, Richards AL. Development of quantitative real-time PCR assays to detect Rickettsia typhi and Rickettsia felis, the causative agents of murine typhus and flea-borne spotted fever. Mol Cell Probes. 2007;21:17-23. http://dx.doi.org/10.1016/j.mcp.2006.06.002

32. Carl M, Tibbs CW, Dobson ME, Paparello S, Dasch GA. Diagnosis of acute typhus infection using the polymerase chain reaction. J Infect Dis. 1990;161:791-3. http://dx.doi.org/10.1093/infdis/161.4.791

33. Bouyer DH, Stenos J, Crocquet-Valdes P, Moron CG, Popov VL, Zavala-Velazquez JE, et al. Rickettsia felis: molecular characterization of a new member of the spotted fever group. Int J Syst Evol Microbiol. 2001;51:339-47. http://dx.doi.org/10.1099/00207713-51-2-339
34. Maina AN, Fogarty C, Krueger L, Macaluso KR, Odhiambo A, Nguyen K, et al. Rickettsial infections among Ctenocephalides felis and host animals during a flea-borne rickettsioses outbreak in Orange County, California. PLoS One. 2016;11:e0160604. http://dx.doi.org/10.1371/journal. pone. 0160604

Address for correspondence: Zeeshan Afzal, McAllen Family Medicine Residency Program, University of Texas Rio Grande Valley, Family and Preventive Medicine, 205 East Toronto Ave, McAllen, TX 78503, USA; email: drzeeshanafzal@gmail.com

\section{July 2013: Vectorborne Infections}

- Transmission of Streptococcus equi Subspecies zooepidemicus Infection from Horses to Humans

- Travel-associated IIIness Trends and Clusters, 2000-2010

- Quantifying Effect of Geographic Location on Epidemiology of Plasmodium vivax Malaria

- Mutation in Spike Protein Cleavage Site and Pathogenesis of Feline Coronavirus
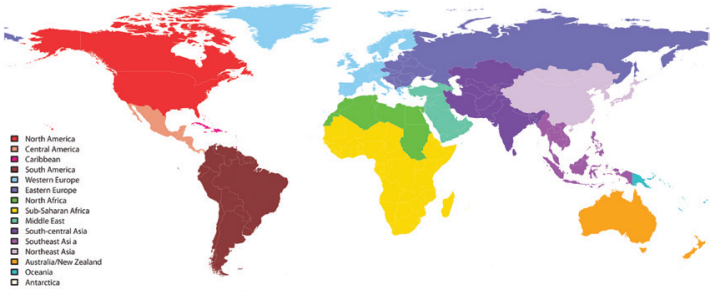

- Pneumococcal Serotypes before and after Introduction of Conjugate Vaccines, United States, 1999-2011

- Influence of Pneumococcal Vaccines and Respiratory Syncytial Virus on Alveolar Pneumonia, Israel

- Avian Metapneumovirus Subgroup C Infection in Chickens, China

- Human Alveolar Echinococcosis in Kyrgyzstan

- Molecular Epidemiologic Source Tracking of Orally Transmitted Chagas Disease, Venezuela

- Unique Clone of Coxiella burnetii Causing Severe Q Fever, French Guiana

- Babesia microti Infection, Eastern Pennsylvania, USA

- Reemergence of Chikungunya Virus in Bo, Sierra Leone

- Novel Bartonella Agent as Cause of Verruga Peruana

- Schmallenberg Virus among Female Lambs, Belgium, 2012

- Psychrobacter arenosus Bacteremia after Blood Transfusion, France
- Ciprofloxacin-Resistant Campylobacter spp. in Retail Chicken, Western Canada

- Asynchronous Onset of Clinical Disease in BSEInfected Macaques

- Prevalence of Nontuberculous Mycobacteria in Cystic Fibrosis Clinics, United Kingdom, 2009

- Reducing Visceral Leishmaniasis by Insecticide Impregnation of Bed-Nets, Bangladesh

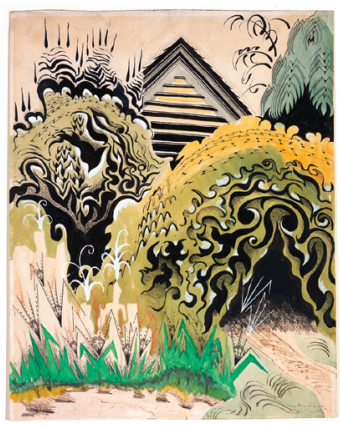

- Genetic Variants of Orientia tsutsugamushi in Domestic Rodents, Northern China

- Undetected Multidrug-Resistant Tuberculosis Amplified by First-line Therapy in Mixed Infection

- Clinical Findings for Early Human Cases of Influenza A(H7N9) Virus Infection, Shanghai, China

- Multidrug-Resistant Atypical Variants of Shigella flexneri in China

- MDR TB Transmission, Singapore

- Human Infection with Marten Tapeworm

- Campylobacter jejuni in Hospitalized Patients with Diarrhea, Kolkata, India



